\title{
RIQUEZA Y DIVERSIDAD DE ÁRBOLES DEL BOSQUE TROPICAL caducifolio en la Depresión Central de Chiapas
}

\author{
Ana Guadalupe Rocha-Loredo, Neptalí Ramírez-Marcial ${ }^{1}$ y Mario González-Espinosa \\ Departamento de Ecología y Sistemática Terrestres, El Colegio de la Frontera Sur \\ ${ }^{1}$ Autor para correspondencia: nramirezm@ecosur.mx
}

\begin{abstract}
Resumen: Se evaluó la diversidad de árboles contenida en 96 parcelas circulares de 0.1 ha, distribuidas en un intervalo altitudinal entre 441 y 1,740 m en la Depresión Central de Chiapas. Las parcelas se clasificaron mediante un análisis de agrupamiento jerárquico y se ordenaron a lo largo de un gradiente ambiental por medio de un análisis de escalamiento multidimensional no métrico (NMDS). La clasificación permitió reconocer cinco asociaciones vegetales, tres de ellas correspondientes al bosque tropical caducifolio (BTC) y dos a los bosques de Quercus. La riqueza regional está representada por un total de 233 especies, en 152 géneros y 57 familias botánicas. La contribución de las parcelas a la diversidad total fue mayor en las asociaciones del bosque tropical caducifolio que en las de Quercus. La composición de especies estuvo dominada por individuos de etapas sucesionales tardías en las asociaciones del BTC (40-56\%), en tanto que en los bosques de Quercus la mayor contribución fue de especies secundarias (46-54\%). La ordenación (NMDS) mostró una relación significativa de las parcelas con 10 de 12 variables climáticas, de las cuales el promedio anual de la temperatura media del mes más caliente $\left(r^{2}=0.74, P<0.001\right)$ y la precipitación media anual $\left(r^{2}=0.85, P<0.001\right)$ fueron las más importantes. A pesar de que en la Depresión Central de Chiapas persiste menos del $2 \%$ de la cobertura forestal, la diversidad de árboles es relativamente alta, debido a la heterogeneidad ambiental que promueve el recambio de especies a nivel regional.
\end{abstract}

Palabras clave: Asociaciones vegetales, heterogeneidad, evapotranspiración real, Quercus NMDS, sucesión ecológica.

\begin{abstract}
Tree diversity in the Central Depression of Chiapas was evaluated through 96 circular plots ( 0.1 ha each) distributed along to altitudinal range between 441 and $1740 \mathrm{~m}$. Hierarchical cluster analysis helped to identify five plant associations; three of them corresponds to the tropical dry forests (TDF), and two Quercus forest. Regional richness included a total of 233 species, 152 genera and 57 botanical families. The contribution of local plots to the total diversity was highest in the TDF associations than in the Quercus forest associations. Species composition was dominated by individuals of late successional stages in the TDF (40-56\%), while greatest contribution was secondary species in the Quercus forests (46-54\%). Ordination showed a significant correlation plots with 10 of 12 climatic variables, which the average annual mean temperature of warmest month $\left(r^{2}=0.74, P\right.$ $<0.001)$ and mean annual precipitation $\left(r^{2}=0.85, P<0.001\right)$ were the most important. Although the Central Depression of Chiapas remains less than $2 \%$ of its forest cover, tree diversity is relatively high due to environmental heterogeneity which promotes species turnover at the regional level.
\end{abstract}

Key words: Actual evapotranspiration, Plant associations, environment heterogeneity, NMDS ordination, Quercus, succession.

$\mathbf{S}^{\mathrm{c}}$ considera que Chiapas es la segunda entidad federativa de México con mayor diversidad florística (Breedlove, 1981), aunque regiones como la Depresión Central (DCCh) no han sido completamente descritas en su composición florística y estructura (Reyes-García y Sousa, 1997). Históricamente la vegetación dominante en la región corresponde a la del Bosque Tropical Caducifolio (BTC, sensu Rzedowski, 1978) y poco se han estudiado las relaciones florísticas con otros tipos de vegetación con los cuales comparte condicio- nes ambientales similares, como un periodo más o menos prolongado de sequía estacional (4-6 meses). Ricker et al. (2007) estimaron que aunque para el año 2000 este tipo de vegetación era el más representado (10.8\% de la superficie forestal del país), al mismo tiempo era de los más afectados por la deforestación a nivel nacional. Las cifras indican que poco menos del $30 \%$ de la cobertura original aun persiste en el país, principalmente a lo largo de la vertiente del Pacífico (Trejo y Dirzo, 2000, Ricker et al., 2007). La condición del 
BTC es aún más crítica en la DCCh, pues estimaciones indican que en la actualidad persiste menos del 5\% de cobertura forestal original (R. Vaca-Genuit, datos no publicados). Aún en esta condición de alta fragmentación, la vegetación actual incluye varias asociaciones distribuidas a lo largo de distintos gradientes climáticos, edáficos, fisiográficos y de disturbio.

La asociación es la unidad conceptual base de la clasificación fitosociológica, que expresa la composición florística general de un conjunto de comunidades vegetales homogéneos estrechamente relacionadas con una determinada región (Font Quer, 2001) y representan grupos florísticos basados en la dominancia de las especies vegetales sensu Beard (1955). Algunas de las comunidades más reconocidas dentro de la DCCh, se corresponden parcial o totalmente con la Selva Baja Caducifolia, Selva Mediana Subcaducifolia, Bosque de Pino-Encino, Encinares (Miranda y Hernández, 1963) o Bosque de Quercus (Rzedowski, 1978). Esta dificultad para precisar los límites entre comunidades de los bosques secos ya han sido señalados con anterioridad (por ej., Pennington et al., 2009; Pérez-García et al., 2009) y reflejan el limitado esfuerzo de muestreo en la región.

En el estudio de los patrones de distribución de la diversidad, se han generado hipótesis que postulan que el principal factor que controla los gradientes de riqueza de especies a nivel global resulta directa o indirectamente de gradientes climáticos (Currie y Paquin, 1987; Stephenson, 1990; O’Brien, 1993; Francis y Currie, 2003). A nivel local pueden influir los factores edáficos (Huston, 1993), o factores microclimáticos en gradientes de luz y temperatura (Godefroid et al., 2006), o la interacción entre gradientes de intensidad de competencia y disturbio (Huston, 1979). Se ha propuesto que otras variables climáticas derivadas de la temperatura y la precipitación pueden ofrecer una explicación con bases más biológicas, como la evapotranspiración real (Wright, 1983; González-Espinosa et al., 2005; 2008) o la evapotranspiración potencial (Palmer y Havens, 1958; Trejo y Dirzo, 2002). En el presente estudio se analizaron los efectos de algunas variables ambientales a nivel regional para explicar la riqueza y diversidad de árboles a nivel local y de toda la región del centro de Chiapas.

Por otro lado, la necesidad de interpretar las respuestas ecofisiológicas de las especies con respecto al ambiente, ha llevado a proponer su clasificación en grupos funcionales, independientemente de su clasificación taxonómica. Las especies se agrupan de acuerdo con su funcionalidad y atributos relevantes con su capacidad de establecimiento y crecimiento bajo condiciones ambientales específicas (Ramírez-Marcial et al., 2008). En Chiapas ha sido posible reconocer grupos funcionales para condiciones ambientales y (o) de disturbio específicas de temperatura y precipitación en áreas de bosque de pino-encino y mesófilo de montaña (por ej., González-Espinosa et al., 1997; Ramírez-Marcial et al., 2005; 2008). No tenemos conocimiento de un inten- to similar para las especies del bosque tropical caducifolio y asociaciones relacionadas, aunque recientemente se ha identificado la necesidad de incorporar el enfoque sucesional en los estudios de los bosques tropicales secos (Quezada et al., 2009). Un método que utiliza el estado sucesional de una especie como una buena aproximación de la diversidad funcional de una comunidad vegetal, nos permite entender aspectos más dinámicos de la comunidad y complementa lo que el análisis basado solamente en la riqueza de especies (Rozza et al., 2007). De acuerdo con lo anterior, en este estudio se analiza la contribución de tres grupos de especies con distinta afinidad sucesional: pioneras, secundarias iniciales y secundarias tardías (Rozza et al., 2007), dentro de las asociaciones identificadas y se discuten las implicaciones de ello en relación con los patrones de perturbación de la vegetación en la región de estudio.

\section{Materiales y métodos}

Área de estudio. La Depresión Central de Chiapas (DCCh) se ubica entre los $17^{\circ} 59^{\prime}-14^{\circ} 32^{\prime}$ de latitud norte y $90^{\circ}$ 22' - $94^{\circ} 14^{\prime}$ de longitud oeste (Figura 1). Comprende más de $280 \mathrm{~km}$ de largo y hasta $70 \mathrm{~km}$ de ancho (aproximadamente $9000 \mathrm{~km}^{2}$ ), con variaciones altitudinales que van desde los 400-1,500 msnm (Müllerried, 1957). La región está completamente rodeada por áreas montañosas húmedas y cálidas en transición con zonas boscosas más frías y secas que le proporcionan una clara y franca zonación respecto a otras regiones donde se presenta el BTC. Los estratos geológicos corresponden en su mayoría a calizas marinas y pizarras del mesozoico. Los suelos son fértiles en general, ya que la mayoría son de origen aluvial, profundos, aunque en las zonas de lomeríos los suelos son delgados y pedregosos (INEGI, 2000). Los tipos de vegetación original, dominados por el Bosque Tropical Caducifolio (Rzedowski, 1978) o Selva Baja Caducifolia y mediana subcaducifolia

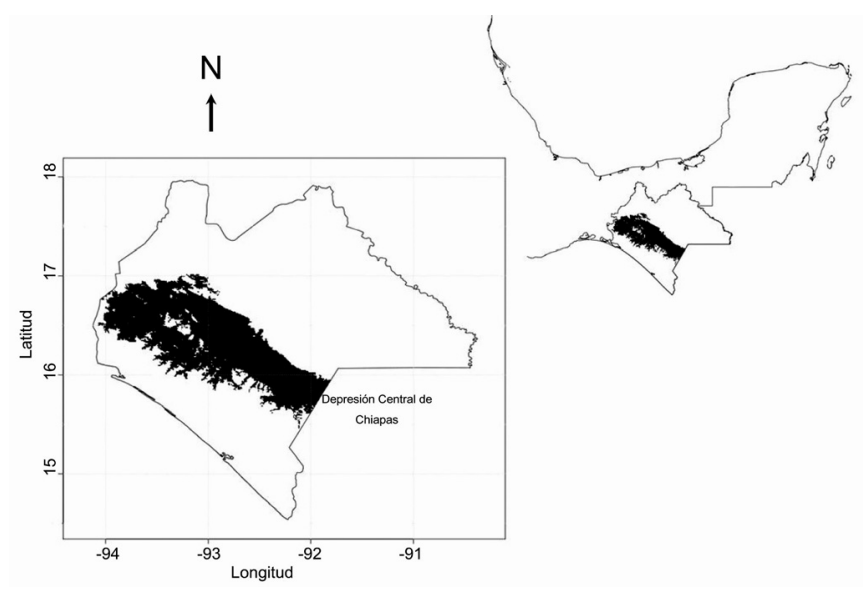

Figura 1. Ubicación geográfica de la Depresión Central de Chiapas, México. 
(Miranda y Hernández-X., 1963), han sido sustituidos en su mayoría por vegetación secundaria debido al pastoreo, el manejo del fuego, la construcción de embalses (La Angostura y Chicoasén) y la apertura de la tierra al cultivo y centros de población humana (INEGI, 2000). Únicamente en ciertas áreas protegidas como cañadas y zonas con alta pendiente se presentan reductos de vegetación con menor evidencia de la influencia humana.

En la región se presenta un régimen de lluvias de verano, de carácter convectivo que definen dos tipos de clima: cálido subhúmedo $\left(\mathrm{Aw}_{0}(\mathrm{w}) \mathrm{igw}\right.$ ") con temperatura media anual de $22.8-25.8^{\circ} \mathrm{C}$; la precipitación pluvial promedio varía entre 660 y $1051 \mathrm{~mm}$ anuales. El otro clima es el semicálido subhúmedo $\left(\mathrm{Aw}_{2}(\mathrm{w})\right.$ igw") que se encuentra en los declives de las cadenas montañosas adyacentes al valle central; la precipitación varía entre 1,110 y $1,267 \mathrm{~mm}$ anuales y temperatura debajo de los $23{ }^{\circ} \mathrm{C}$ (García, 1988). La mayor precipitación se concentra en los meses de junio a septiembre, y entre julio y agosto disminuye (canícula), aumentando nuevamente a finales de agosto. En la DCCh y zonas adyacentes la lluvia declina a partir de octubre y llega a ser muy escasa entre noviembre y abril.

Muestreo de la vegetación. Se analizaron la composición florística y abundancia de árboles contenida en 96 parcelas circulares de 0.1 ha cada una (sensu Ramírez-Marcial et al., 2001). Las parcelas se localizaron en diferentes localidades ubicadas entre 441-1740 m de altitud. En cada parcela se registró la ocurrencia, abundancia y el diámetro de todos los individuos $\geq 10 \mathrm{~cm}$ de diámetro a la altura del pecho (DAP). La estructura de la vegetación se analizó con base en los valores relativos de la densidad y área basal de los árboles para obtener un índice de valor de importancia (Müller-Dombois y Ellenberg, 1974). Durante el muestreo en campo, se elaboró un catálogo itinerante consistente en muestras vegetativas o reproductivas de cada una de las morfoespecies. Dichas muestras se colocaron en dos folios de papel secante con un número de identificación consecutivo conforme se iban incorporando en el muestreo. Cuando fue posible, se realizó la colecta botánica de aquellos ejemplares con estructuras reproductivas y conjuntamente con el material del catálogo itinerante se determinó mediante cotejo con ejemplares en el herbario de ECOSUR, Unidad San Cristóbal. Los nombres taxonómicos fueron corroborados con la base de datos del Jardín Botánico de Missouri W3TROPICOS $<w w w . t r o p i c o s . o r g>$.

Análisis de datos. Se construyó una primera matriz de datos con la densidad y área basal de los individuos de cada especie en las 96 parcelas; la otra matriz consistió de 12 variables ambientales. Las variables climáticas se tomaron de Worldclim-Bioclim <www.worldclim.org> con una resolución espacial de $1 \mathrm{~km}^{2}$, que se generan por medio de interpolación de datos de estaciones meteorológicas y fueron desarrollados para todo el mundo (Hijmans et al., 2005). La evapotranspiración real anual (ETRA) se calculó para cada parcela de acuerdo con el modelo de Turc (1954): $E T R A=P /\left[0.9+(\mathrm{P} / \mathrm{L})^{2}\right]^{1 / 2}$, donde $L=300+25 T+0.05 T^{3}$, $P=$ precipitación pluvial anual y $T=$ temperatura media anual. La ETRA es considerada como una medida de la cantidad de agua de lluvia disponible a las plantas bajo temperaturas propicias para su crecimiento (Rosenzweig, 1968) y se parte de la premisa que un eje de recursos más amplio (energía, agua, nutrimentos) puede permitir la existencia de un mayor número de individuos, y por tanto, de un mayor número de especies (Wright, 1983; Ricklefs, 1987; Rosenzweig y Abramsky, 1993; Currie et al., 2004).

Con la primera matriz se realizó la clasificación jerárquica de la vegetación mediante un análisis TWINSPAN (Two Way Indicator Species Analysis; Hill, 1979) con el programa PC-ORD versión 4.17 (McCune y Mefford, 1999). Este método se basa en el concepto de que un grupo de sitios (parcelas) puede tener un grupo correspondiente de especies indicadoras. Esta división entre grupos es equivalente a un análisis de correspondencia, donde cada muestra es dividida dicotómicamente en un grupo negativo y positivo que corresponde a sus valores característicos (eigenvalues) del primer eje de la ordenación y define la pertenencia a uno u otro grupo (Leps y Smilauer, 2003). La separación de grupos se decidió después de los tres primeros niveles de corte. Alternativamente al análisis de TWINSPAN, se realizó un análisis de agrupamiento jerárquico, aplicado a una matriz de disimilitud obtenida con los datos de densidad por el método de Ward y procedimiento de agrupamiento promedio sin ponderación (UPGMA), por ser uno de los métodos que introduce menor distorsión en la distancia entre agrupamientos respecto a la matriz de distancia original (Oksanen, 2010). De éste análisis se obtuvo un dendrograma cuya escala vertical (valores entre 0 y 1 ) expresa la disimilitud entre sitios, donde valores cercanos a cero representan mayor similitud (Oksanen, 2010). Para determinar el número de grupos en el dendrograma se utilizó la función "hclust" de la rutina vegan para desplegar gráficamente el número de grupos (Oksanen, 2010) operada desde el programa $R$ (R Development Core Team, 2010) y posteriormente se inspeccionó su correspondencia con el resultado del análisis de TWINSPAN. Para los cinco grupos de vegetación, se construyó una curva de acumulación de especies-área.

Para la ordenación indirecta de las parcelas, se realizó un análisis de escalamiento no métrico multidimensional, NMDS (Non-metric Multidimensional Distance Scaling) el cual utiliza la matriz de disimilitud entre parcelas; éste método se considera uno de los más efectivos para ordenar datos ecológicos al no requerir el supuesto de relación lineal entre las variables. Aunque este análisis tiene la desventaja de encontrar más de una solución, es necesario realizar un procedimiento de reiteración hasta encontrar el valor de tensión (stress) más pequeño. Para ello se utilizó un proce- 
dimiento automatizado incluido en la función "metaMDS" dentro de la rutina MASS operada desde el programa R. Finalmente, se graficaron los dos primeros ejes de la ordenación y sobre éstos se sobrepusieron los grupos obtenidos en el dendrograma.

El método de interpretación de los grupos (asociaciones) es ajustar los vectores de las variables ambientales sobre los ejes de ordenación con la función "envfit". Para tal fin se usaron las 12 variables ambientales para obtener un valor de correlación y significancia de cada una a lo largo de los ejes de la ordenación. El valor de significancia se basa en permutaciones aleatorias de los datos y si estos tienen un mayor ajuste que el de los vectores, estos se consideran no significativos (Oksanen, 2010).

Para cada parcela se calculó la diversidad con el índice de Shannon-Wiener ( $H$, base logaritmo natural) y el índice de uniformidad $(H / \ln (S)$, donde $S=$ la riqueza de especies (Rosenzweig, 1995). Para evaluar la contribución de cada parcela a la diversidad regional, seguimos las recomendaciones de Lu et al. (2007) para diferenciar la diversidad local dentro y entre unidades de muestreo. Para ello utilizamos un procedimiento de repartición aditiva de la diversidad y distintividad (exclusividad) de unidades. Finalmente se analizó la diversidad considerando la afinidad sucesional de las especies entre las asociaciones vegetales. Todos los análisis se obtuvieron con la función "contribdiv" contenida en el paquete BiodiversityR (Kindt, 2009).

\section{Resultados}

A nivel regional y considerando 96 parcelas (se excluyó la parcela 44 por errores de captura), se registraron 5327 individuos mayores de $10 \mathrm{~cm}$ de DAP, pertenecientes a 233 especies, 152 géneros y 56 familias botánicas (Apéndice 1). Las familias con más especies fueron Fabaceae (33 especies), Euphorbiaceae (14), Malvaceae (11) y Rubiaceae (10). De las familias restantes, 19 de ellas estuvieron representadas por una sola especie (Apéndice 1).

Debido a la heterogeneidad ambiental incorporada en el muestreo, se registraron claras diferencias florísticas y estructurales entre los sitios de estudio. El análisis de agrupación jerárquica permitió reconocer cinco asociaciones de vegetación con base en la disimilitud de las especies dominantes entre parcelas con base en sus valores de importancia (Figura 2). Tres de estas asociaciones corresponden a las comunidades del Bosque Tropical Caducifolio cuyas parcelas se ubicaron a menor altitud ( $<900 \mathrm{~m}$ ) y dos a los bosques de pino-encino, generalmente ubicadas a mayor elevación $(>1,000 \mathrm{~m})$.

Todas las asociaciones están representadas por diferente número de parcelas y todas ellas mostraron un aumento en el número acumulativo de especies con el incremento en el número de parcelas por asociación (Figura 3). El número promedio de especies por parcela varió entre 13 y 18 especies para las asociaciones del BTC y contrastó con la ri-

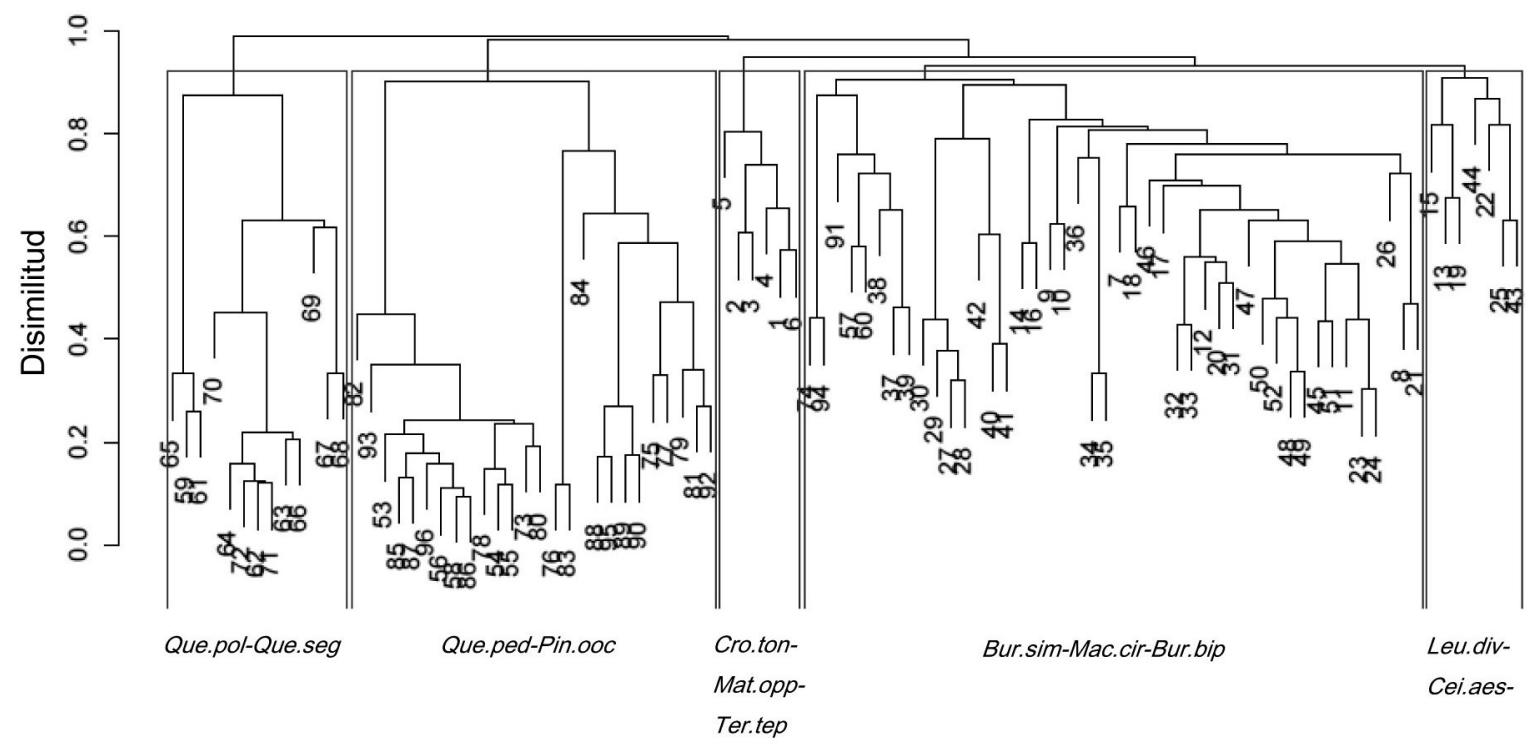

\section{Asociaciones}

Figura 2. Dendrograma del agrupamiento jerárquico de las 96 parcelas de muestreo en la Depresión Central de Chiapas. Los paneles abiertos insertos en el dendrograma delimitan las cinco asociaciones identificadas a partir de su disimilitud florística: Mat.opo-Ter.tep = Mataiba oppositifolia-Ternstroemia tepezapote, Bur.sim-Coc.vit = Bursera simaruba-Cochlospermun vitiifolium, Leu.div-Cei.aes $=$ Leucaena diversifolia-Ceiba esculifolia, Que.ped-Pin.ooc = Quercus peduncularis-Pinus oocarpa, Que.pol-Que.seg = Quercus polymorpha-

Quercus segoviensis. 


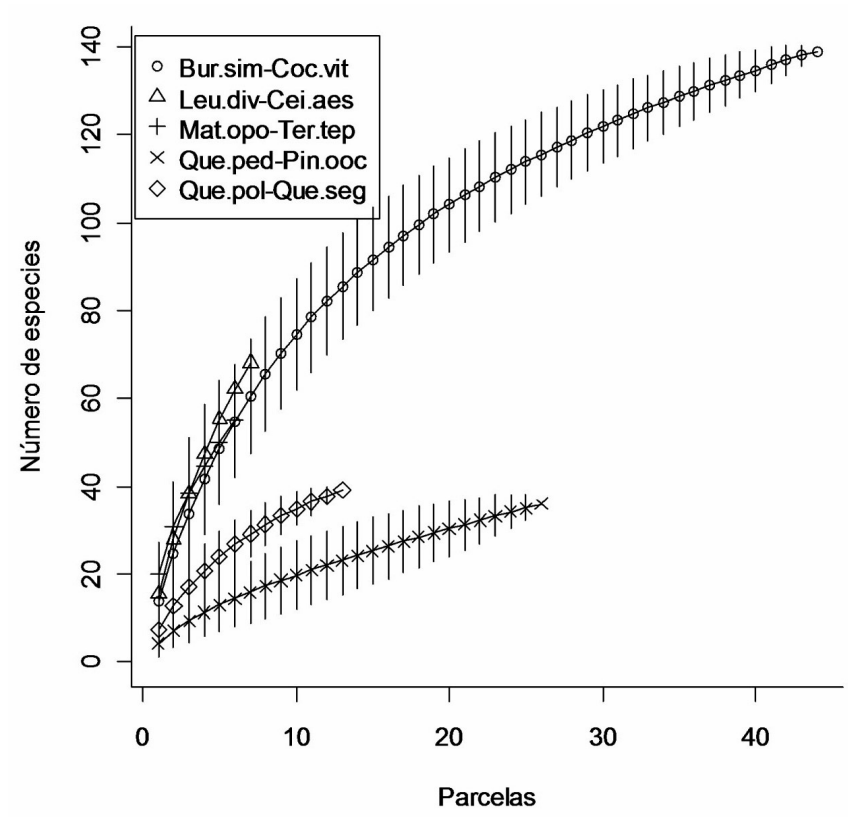

Figura 3. Curvas de acumulación de especies en función del número de parcelas para cada una de las cinco asociaciones de vegetación identificadas por el análisis de clasificación jerárquica. Cada curva representa un muestreo aleatorio con reemplazo y las líneas verticales expresan un valor promedio + 1 E.E.

queza por parcela registrada en las dos asociaciones de los bosques de Quercus (4-6 especies por parcela; Cuadro 1) La diversidad de árboles fue estadísticamente diferente entre las asociaciones (índice de Shannon: $F_{4,91}=43.2, P \leq 0.001$ ). La asociación de Matayba oppositifolia (A. Rich.) Britton Ternstroemia tepezapote Schltdl. \& Cham., fue la que mostró la mayor diversidad de Shannon-Wiener y uniformidad, seguida de la asociación de Leucaena diversifolia (Schltdl.) Benth. - Ceiba aesculifolia (Kunth) Britten \& Baker f., Bursera simaruba (L.) Sarg. - Cochlospermum vitiifolium (Willd.) Spreng., Quercus polymorpha Schltdl. \& Cham.

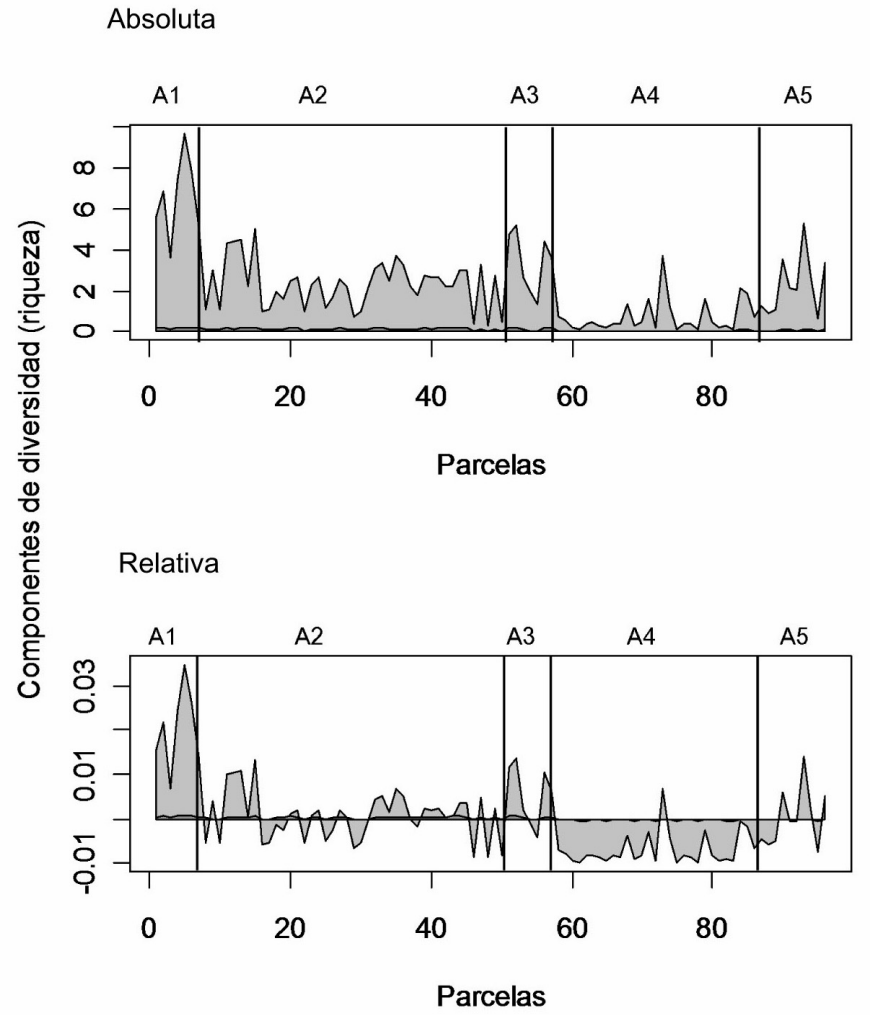

Figura 4. Componentes de diversidad (riqueza de especies), sensu Lu et al. (2007). El panel superior expresa la contribución absoluta de las parcelas individuales y de las asociaciones a la diversidad regional. El panel inferior representa la proporción de la riqueza regional contenida a nivel de cada parcela. Valores positivos indican que la contribución de una parcela es superior al promedio de todas las parcelas, en consecuencia, valores negativos indican una menor contribución individual respecto al promedio total. A1= Mataiba oppositifolia-Ternstroemia tepezapote, A2 = Bursera simaruba-Cochlospermun vitiifolium, A3 = Leucaena diversifolia-Ceiba esculifolia, A4 = Quercus peduncularis-Pinus oocarpa, A5 = Quercus polymorpha-Quercus segoviensis.

Cuadro 1. Atributos florísticos y ambientales de la vegetación leñosa en la Depresión Central de Chiapas. Asociaciones: Mat.opo-Ter.tep = Mataiba oppositifolia-Ternstroemia tepezapote, Bur.sim-Coc.vit = Bursera simaruba-Cochlospermun vitiifolium, Leu.div-Cei.aes = Leucaena diversifoliaCeiba esculifolia, Que.ped-Pin.ooc = Quercus peduncularis-Pinus oocarpa, Que.pol-Que.seg = Quercus polymorpha-Quercus segoviensis.

\begin{tabular}{|c|c|c|c|c|c|}
\hline & $\begin{array}{c}\text { Mat.opo- } \\
\text { Ter.tep }\end{array}$ & $\begin{array}{l}\text { Bur.sim- } \\
\text { Coc.vit }\end{array}$ & $\begin{array}{l}\text { Leu.div- } \\
\text { Cei.aes }\end{array}$ & $\begin{array}{l}\text { Que.ped- } \\
\text { Pin.ooc }\end{array}$ & $\begin{array}{l}\text { Que.pol- } \\
\text { Que.seg }\end{array}$ \\
\hline Número de parcelas & 6 & 44 & 7 & 26 & 13 \\
\hline Riqueza por parcela (media \pm E.E) & $18 \pm 1.4$ & $13 \pm 0.5$ & $14.5 \pm 1.3$ & $4 \pm 0.7$ & $6 \pm 1.0$ \\
\hline Riqueza acumulada & 57 & 154 & 79 & 37 & 39 \\
\hline Indice de Shannon-Wiener (media \pm E.E) & $2.6 \pm 0.19$ & $2.1 \pm 0.07$ & $2.3 \pm 0.017$ & $0.84 \pm 0.09$ & $0.98 \pm 0.13$ \\
\hline Índice de Uniformidad $(\mathrm{H} / \ln (\mathrm{S}))$ & $0.87 \pm 0.06$ & $0.83 \pm 0.02$ & $0.86 \pm 0.05$ & $0.6 \pm 0.03$ & $0.54 \pm 0.04$ \\
\hline Altitud mínima y máxima (m) & 926-1022 & $441-1231$ & $1225-770$ & $704-1373$ & 810-1064 \\
\hline Temperatura media anual promedio $\left({ }^{\circ} \mathrm{C}\right)$ & 22.5 & 23.7 & 18.6 & 22.8 & 22.9 \\
\hline Precipitación media anual (mm) & 960 & 1045 & 990 & 1135 & 999 \\
\hline
\end{tabular}


Cuadro 2. Contribución de la afinidad sucesional (número de especies y su porcentaje del total dentro de la asociación) para las cinco comunidades vegetales registradas en la Depresión Central de Chiapas. La clasificación según Rozza et al. (2007): Pioneras, son intolerantes a la sombra, colonizadoras de grandes claros, forman bancos de semillas en el suelo, con un crecimiento muy rápido, ciclos de vida corto y de madera blanda; Secundarias iniciales, semejantes a las especies pioneras, pero que no forman bancos de semillas en el suelo y presentan ciclos de vida un poco más largo y su establecimiento ocurre después que las pioneras dejan el lugar y Tardías, tienen un ciclo de crecimiento más lento por su mayor longevidad, madera dura, plántulas y juveniles tolerantes a la sombra y frutos carnosos o secos grandes, generalmente dispersadas por vertebrados. Nombre de las asociaciones como en la leyenda del Cuadro 1.

\begin{tabular}{lccccc}
\hline \multicolumn{5}{c}{ Asociaciones } \\
\cline { 2 - 6 } $\begin{array}{l}\text { Afinidad } \\
\text { sucesional }\end{array}$ & $\begin{array}{c}\text { Mat.opo- } \\
\text { Ter.tep }\end{array}$ & $\begin{array}{c}\text { Bur.sim- } \\
\text { Coc.vit }\end{array}$ & $\begin{array}{c}\text { Leu.div- } \\
\text { Cei.aes }\end{array}$ & $\begin{array}{c}\text { Que.ped- } \\
\text { Pin.ooc }\end{array}$ & $\begin{array}{c}\text { Que.pol- } \\
\text { Que.seg }\end{array}$ \\
\hline Pioneras & $8(14)$ & $24(15.5)$ & $19(24.5)$ & $11(29.7)$ & $7(17.9)$ \\
$\begin{array}{l}\text { Secundarias } \\
\text { iniciales }\end{array}$ & $17(29.8)$ & $48(31.1)$ & $25(31.6)$ & $17(45.9)$ & $21(53.8)$ \\
Tardías & $32(56.4)$ & $68(44.1)$ & $32(40.5)$ & $8(21.6)$ & $10(25.6)$ \\
$\begin{array}{l}\text { No } \\
\text { determinadas }\end{array}$ & & $14(9.1)$ & $3(3.7)$ & $1(2.7)$ & $1(2.5)$ \\
\hline
\end{tabular}

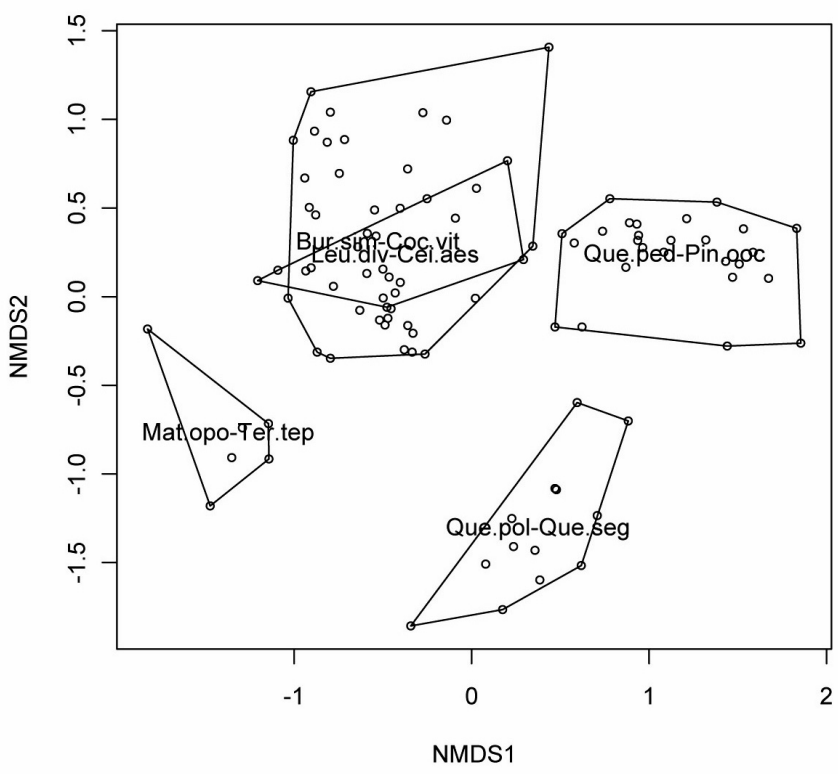

Figura 5. Diagrama de ordenación de 96 parcelas de muestreo de la vegetación leñosa en la Depresión Central de Chiapas a lo largo de los dos primeros ejes de ordenación mediante escalamiento no métrico multidimensional (NMDS). Los polígonos corresponden a las cinco asociaciones vegetales obtenidas previamente con el análisis de agrupación. Mat.opo-Ter.tep = Mataiba oppositifolia-Ternstroemia tepezapote, Bur.sim-Coc.vit = Bursera simaruba-Cochlospermun vitiifolium, Leu.div-Cei.aes = Leucaena diversifolia-Ceiba esculifolia, Que.ped-Pin.ooc = Quercus peduncularis-Pinus oocarpa, Que.pol-Que.seg = Quercus polymorphaQuercus segoviensis.
Cuadro 3. Valores de correlación para los dos primeros ejes del análisis de escalamiento multidimensional no métrico (NMDS) para las 12 variables ambientales consideradas. En negritas las variables con la mayor correlación.

\begin{tabular}{lrrrrr}
\hline Variables ambientales & NMDS1 NMDS2 & $r^{2}$ & $\operatorname{Pr}(>\mathrm{r})$ \\
\hline Temperatura media anual & -0.20 & -0.98 & 0.69 & $<0.001$ \\
Rango de la T media diurna & 0.51 & -0.86 & 0.08 & 0.016 \\
T máxima del mes más caliente & -0.26 & -0.97 & $\mathbf{0 . 7 4}$ & $<0.001$ \\
T mínima del mes más frío & -0.28 & -0.96 & 0.53 & $<0.001$ \\
Rango de la T anual & -0.20 & -0.98 & 0.29 & $<0.001$ \\
Precipitación media anual & 0.83 & -0.55 & $\mathbf{0 . 8 5}$ & $<0.001$ \\
Prec del mes más humedo & 0.78 & -0.62 & 0.30 & $<0.001$ \\
Prec del mes más frío & -0.05 & 1.00 & 0.54 & $<0.001$ \\
Diferencia de la TT máxima anual & -0.95 & -0.31 & 0.49 & $<0.001$ \\
Diferencia de la TT diaria & 0.46 & -0.89 & 0.16 & $<0.001$ \\
Meses de sequia & 0.63 & -0.78 & 0.01 & 0.59 \\
Evapotranspiración real & 0.64 & -0.77 & 0.28 & $<0.001$ \\
anual (ETR) & & & & \\
\hline
\end{tabular}

- Quercus segoviensis Liebm., y por último la asociación de Quercus peduncularis Née - Pinus oocarpa Schiede ex Schltdl. (Cuadro 1).

$\mathrm{Al}$ analizar la contribución de la diversidad local (a nivel de parcelas) a la diversidad regional (total de las 96 parcelas) se detectó que cada parcela contribuye de manera considerable a la diversidad local, al contener varias especies exclusivas. El coeficiente de diferenciación en escala de 0-1, fue de 0.949 y según Lu et al. (2007) esto significa que la diversidad regional está repartida en todos los sitios o parcelas. La figura 4, muestra como en términos absolutos y relativos la contribución de cada parcela dentro de las cinco asociaciones vegetales, donde resalta que la asociación de Matayba oppositifolia-Ternstroemia tepezapote es la que tiene mayor contribución de especies exclusivas a la diversidad total, en tanto que la de menor contribución corresponde a la de Quercus peduncularis- Pinus ocarpa, expresada por los valores relativos negativos en el panel inferior de la figura 4 y que se interpreta que su contribución como asociación es inferior al promedio de todas las parcelas.

En general, la composición de las especies por su afinidad sucesional fue significativamente diferente entre las asociaciones (Pearson $\chi^{2}=19.82,8$ g.l., $P=0.011$ ). La composición de especies estuvo dominada por individuos de etapas sucesionales tardías en las tres asociaciones del BTC (40-56\%), en tanto que en los bosques de Quercus la mayor contribución fue de especies secundarias (46-54\%; Cuadro 2).

El análisis de ordenación mediante (NMDS) mostró una relativa correspondencia entre las asociaciones identificadas por el análisis de agrupamiento jerárquico. El coeficiente de bondad de ajuste contenido en los tres ejes de la ordenación ambiental (Stress $=16.16$ ), permitió ubicar a las cinco asociaciones anteriormente reconocidas por el 
análisis de agrupación (Figura 5). Sin embargo, fue notable que la asociación de Leucaena-diversifolia-Ceiba aesculifolia está anidada a la de Bursera simaruba-Cochlospermum vitifolium. Las dos asociaciones del bosque de Quercus se localizaron a la derecha del primer eje, mientras que las tres del BTC se ubicaron a la izquierda del mismo eje. De las 12 variables ambientales incluidas en el análisis 10 de ellas resultaron significativas en predecir el ordenamiento de las parcelas (Cuadro 3). De éstas variables significativas, la precipitación media anual fue la más altamente correlacionada con el primer eje y la temperatura máxima del mes más caliente con el segundo eje de la ordenación (Cuadro 3).

\section{Discusión}

La vegetación de la Depresión Central de Chiapas a pesar de ser considerada convencionalmente como una sola identidad florística (por ej., Reyes-García y Sousa, 1997; Rzedowski, 1978; Breedlove, 1981), en realidad es un conjunto de diversas asociaciones de vegetación representadas en los cinco grupos definidos por el análisis de agrupación. Hay que recordar las analogías y similitudes fisonómicas entre diferentes comunidades vegetales como la Selva Baja Caducifolia, Selva Mediana Subcaducifolia, Bosque de Pino-Encino, e incluso Encinares (Miranda y Hernández-X., 1963) y Bosque de Quercus (Rzedowski, 1978). Varios autores han hecho distintos esfuerzos de clasificación de la vegetación del bosque tropical caducifolio y su delimitación geográfica en México; por ejemplo Rzedowski (1978), Trejo y Dirzo (2002), Becerra (2005) y para bosques secos de Mesoamérica (Barrance et al., 2009) y del continente americano (Pennington et al., 2009). Las asociaciones vegetales obtenidas en este estudio corresponden geográficamente a la clasificación de estos autores. Aunque en nuestro caso, se han incluido dos asociaciones cuya composición está dominada por especies con afinidad boreal (principalmente los bosques de Quercus y Pinus-Quercus), climáticamente comparten los mismos atributos del bosque seco y este criterio ha sido utilizado en sentido amplio para denotar a los bosques tropicales secos estacionales (Pennington et al., 2009).

En términos florísticos, la familia con mayor número de especies en la vegetación muestreada fue Fabaceae, lo cual coincide con los resultados de otros estudios en bosques secos en otras regiones en México a nivel local como Chamela en Jalisco (Lott et al., 1987), Colima (Salinas-Melgoza, 2007), la cuenca del Balsas en Guerrero (Pineda-García et $a l ., 2007)$, la sierra de Nizanda en Oaxaca (Pérez-García et al., 2001). Sin embargo, en términos de la contribución del Índice de Valor de Importancia (IVI), la familia Fagaceae fue la más representativa (IVI $=38.3 \%$ ) dentro de las asociaciones ubicadas a mayor altitud. Algunos géneros como Quercus y Bursera pertenecn a diferente origen fitogeográfico que permiten reconocer fisonómicamente a los bosques secos de montaña (dominados por Quercus) y los bosques cálido-secos (representados por Bursera), pero en varias parcelas ambos géneros estuvieron presentes compartiendo dominancia, lo cual sugiere que en el muestreo se incluyeron zonas de transición entre el BTC y los bosques templados y estacionales.

Entre las cinco asociaciones obtenidas se encontró que la diversidad de especies arbóreas fue mayor en la asociación Mat.opo-Ter.tep; está formada por varias especies que no aparecen en las demás asociaciones. Por su ubicación, el ambiente es más húmedo y favoreció una mayor representación de especies típicamente encontrados en bosques húmedos y en estadio sucesional climácico, como Nectandra salicifolia, $N$. reticulata, Persea liebmannii y Terminalia oblonga. Esta localidad se encuentra en el límite entre la región cálido-húmeda en la reserva de El Ocote y la región propiamente cálido seca de la Depresión Central de Chiapas. En la asociación Bur.sim-Coc.vit la especie Bursera simaruba fue la más abundante, lo cual concuerda como rasgo característico de las selvas bajas caducifolias de otras regiones de México (Rzedowski, 1978; Pineda-García et al., 2007, Pennington et al., 2009). Esta asociación florísticamente se asocia con el Bosque Tropical Caducifolio, sensu Rzedowki (1978). Por otro lado, la asociación Que.pol-Que. ped representa una zona de transición de los bosques estacionales caducifolios, por un lado los encinares secos a mayores elevaciones y el bosque tropical caducifolio a altitudes menores. Por el régimen climático compartido entre estas asociaciones, se considera que son un tipo de vegetación que se ha ajustado a climas cálido-fríos y resguardan especies de diferentes afinidades geográficas.

Debido a que la diversidad de especies se distribuye de manera diferente entre las distintas unidades de muestreo, se espera que la contribución de dichas unidades a nivel regional tampoco sea uniforme (Lu et al., 2007). La alta complementariedad entre las asociaciones vegetales tiene implicaciones desde el punto de vista de la conservación. Al haber un alto recambio de especies entre las parcelas (como lo sugieren las curvas de acumulación de especies y el análisis de la contribución de la diversidad local a la diversidad regional, Figuras 2 y 4, respectivamente), se explica con ello la necesidad de conservar la mayor cantidad de los fragmentos actuales de bosque en la región, pues cada uno de ellos contribuye con especies exclusivas (Lu et al., 2007). Una alta diversidad de cambio entre unidades de muestreo (diversidad beta) significa que la composición de especies varía de un sitio a otro y que por lo tanto una reserva establecida en un sitio no necesariamente incluirá a las especies que se encuentren en otros sitios (Rodríguez y Arita, 2004). El estudio de Castillo-Campos et al. (2008) sugiere que es necesaria la conservación de bosques secundarios y primarios al complementar la diversidad regional. Esta observación se confirma en este estudio ya que a nivel local hay especies exclusivas no solo a nivel de la asociación, sino a nivel de parcelas, que no se repiten en otros sitios $\mathrm{y}(\mathrm{o})$ asociaciones. 
Por ejemplo ninguna especie estuvo presente en todos los sitios de muestreo, a pesar de que Bursera simaruba se registró en 47 de las 96 parcelas.

La vegetación en la Depresión Central está preponderantemente representada por las especies tardías, principalmente en las tres asociaciones del BTC (40-56\%), en tanto que en los bosques de Quercus la mayor contribución fue de especies secundarias (46-54\%). En el primer caso, este resultado puede interpretarse como que estas comunidades mantienen aún una alta proporción de sus elementos característicos de bosques primarios, que aunque fragmentados, en estos remanentes de vegetación es posible mantener poblaciones de dichas especies. Por el otro lado, la mayor proporción de especies secundarias en ambas asociaciones de los bosques de Quercus indica un mayor efecto antropógeno sobre su composición y estructura, tal y como ha sido señalado para comunidades de pino-encino colindantes en la región de Los Altos de Chiapas (González-Espinosa et al., 2005).

Actualmente, la mayoría de los paisajes de BTC son mosaicos de tierras agrícolas, vegetación secundaria, y parches de vegetación primaria relativamente conservada (CastilloCampos et al., 2008). El constante cambio de uso de suelo resulta en un mosaico complejo a nivel de paisaje, que se ve influenciado por actividades humanas, mismas que no fueron consideradas de manera directa en el presente trabajo. Cuando se mide el disturbio antrópico de manera directa resulta ser vital para la explicación de la distribución de la diversidad arbórea a escalas de paisaje, pero hay pocos estudios que evalúen este factor para el bosque tropical seco, por ejemplo el de Lebrija-Trejos et al. (2008) donde encuentran que sitios más maduros cuentan con una mayor cantidad y densidad de especies.

Aunque se detectaron diferencias significativas en las características climáticas entre las asociaciones, no es posible atribuir de manera definitiva una relación de causaefecto entre el clima y la diversidad, sin antes incorporar otras variables predictivas como el disturbio. Por ahora solo es posible señalar que la diversidad de la vegetación leñosa tiene relación con la altitud expresada en variaciones en la precipitación y temperatura. Aunque la evapotranspiración real anual (ETRA) se encontró útil para explicar la variación espacial de la diversidad de árboles de Chiapas (González-Espinosa et al., 2004), en el presente estudio esta variable tuvo bajo poder explicativo $(P=0.28$; Cuadro 3$)$, posiblemente debido a que el gradiente para esta variable fue menor en nuestro estudio $(670 \mathrm{~mm}$ a $1049 \mathrm{~mm})$ que en el de González-Espinosa et al. (2004) el cual incluyó una variación de 680 a $1500 \mathrm{~mm}$.

Se concluye que la región de la Depresión Central de Chiapas contiene aun una alta diversidad local, con apreciables cambios en la composición de especies de un sitio a otro. Estos atributos, le confieren por complementariedad una alta diversidad regional. La vegetación actual concentrada en pequeños fragmentos está bajo el efecto combinado de la heterogeneidad ambiental, incluidas variaciones climáticas, topográficas, edáficas y de disturbio antropógeno. Se reconoce una necesidad de continuar el estudio de los bosques secos tropicales del estado, que incorpore no solamente aspectos biológicos, sino socioeconómicos, en especial lo referente a los cambios de uso del suelo y sus efectos sobre la fragmentación y dinámica sucesional de las comunidades remanentes.

\section{Agradecimientos}

Agradecemos a Nathaline Taylor-Aquino, Magdiel LuisSantiago, Abril Valdivieso, Miguel Martínez-Icó, Henry Castañeda-Ocaña quienes participaron en el trabajo de campo. Raúl Vaca ayudó en los análisis geográficos y elaboración de la figura 1. Esta publicación ha sido posible gracias al apoyo proporcionado por la Unión Europea a través del proyecto ReForLan (INCO-DEV-3, Framework Programme 6, contrato No. 032132) y del Fondo Institucional de Fomento Regional para el Desarrollo Científico, Tecnológico y de Innovación (FORDECYT-CONACyT) a través del Convenio 116306: Innovación socioambiental para el desarrollo en áreas de alta pobreza y biodiversidad de la frontera sur de México.

\section{Literatura citada}

Barrance A., Schreckenberg K. y Gordon J. 2009. Conservation through use: lessons from the Mesoamerican dry forest. Overseas Development Institute. Reino Unido.

Beard J.S. 1955. The classification of tropical American vegetation-types. Ecology 36:89-100.

Becerra J.X. 2005. Timing the origin and expansion of the Mexican tropical dry forest. Proceedings of the National Academy of Sciences 102:10919-10923.

Breedlove D.E. 1981. Flora of Chiapas. Part I: Introduction to the flora of Chiapas. California Academy of Sciences. EUA.

Castillo-Campos G., Halffter G. y Moreno C.E. 2008. Primary and secondary vegetation patches as contributors to floristic diversity in a tropical deciduous forest landscape. Biodiversity and Conservation 17:1701-1714.

Currie D.J., Mittelbach G.G., Cornell H.V., Field R., Guégan J.F., Hawkins B.A., Kaufman D.M., Kerr J.T., Oberdorff T., O’Brien E. y Turner J.R.G. 2004. Predictions and tests of climate-based hypotheses of broad-scale variation in taxonomic richness. Ecology Letters 7:1121-1134.

Currie D.J. y Paquin V. 1987. Large-scale biogeographical patterns of species richness in trees. Nature 329:326-327.

Font Quer P. 2001. Diccionario de Botánica, $2^{\mathrm{a}}$ edición. Ediciones Península, Barcelona.

Francis A.P. y Currie D. J. 2003. A globally consistent richnessclimate relationship for angiosperms. American Naturalist 161:523-536.

García E. 1988. Modificaciones al sistema de clasificación climática de Köppen. Instituto de Geografía, Universidad Nacional Autónoma de México. México, D.F.

Godefroid S., Rucquoij S. y Koedam N. 2006. Spatial variabili- 
ty of summer microclimates and plant species response along transects within clearcuts in a beech forest. Plant Ecology 185:107-121

González-Espinosa M., Ochoa-Gaona S., Ramírez-Marcial N., y Quintana-Ascencio P.F. 1997. Contexto vegetacional y florístico de la agricultura. En: Parra-Vázquez M.R. y Díaz-Hernández B.M. Eds. Los Altos de Chiapas: agricultura y crisis rural, pp. 85-117. El Colegio de la Frontera Sur. México,

González-Espinosa M., Rey-Benayas J.M., Ramírez-Marcial N., Huston M.A. y Golicher D. 2004. Tree diversity in the northern Neotropics: regional patterns in highly diverse Chiapas, Mexico. Ecography 27:741-756.

González-Espinosa M., Ramírez-Marcial N., Méndez-Dewar G., Galindo-Jaimes L. y Golicher D. 2005. Riqueza de especies de árboles en Chiapas: variación espacial y dimensiones ambientales asociadas a nivel regional. En: González-Espinosa, M., Ramírez-Marcial N., Ruiz Montoya L. Coord. Diversidad biológica en Chiapas, pp. 81-126. Plaza y Valdés, México.

González-Espinosa M., Ramírez-Marcial N. y Rey-Benayas J.M. 2008. Variación espacial de la diversidad arbórea de Chiapas: patrones regionales y jerarquía de factores determinantes. En: Sánchez-Velázquez L.R., Galindo-González J. y Díaz-Fleischer F. Eds. Ecología, Manejo y Conservación de los Ecosistemas de Montaña en México, pp. 147-166. Mundi-Prensa. México.

Hijmans R.J., Cameron S.E., Parra J.L., Jones P.G. y Jarvis A. 2005. Very high resolution interpolated climate surfaces for global land areas. International Journal of Climatology 25:19651978.

Hill M.O. 1979. TWISPAN-A FORTRAN program for arranging multivariate data in an ordered two-way table by classification of the individuals and attributes. Cornell University, Ithaca, Nueva Jersey.

Huston M. 1979. A general hypothesis of species diversity. American Naturalist 113:81-101.

Huston M. 1993. Biological diversity, soils, and economics. Science 262:1676-1680

INEGI [Instituto Nacional de Geografía y Estadística]. 2000. Uso del Suelo y Vegetación 2000 conjunto de datos del ordenamiento territorial estatal. Escala 1:250,000.

Lebrija-Trejos E., Bongers F., Pérez-García E.A. y Meave J.A. 2008. Successional change and resilience of a very dry tropical deciduous forest following shifting agriculture. Biotropica 40:422-431.

Leps J. y Smilauer P. 2003. Multivariate analysis of ecological data using CANOCO. Cambridge University Press, Cambridge, Reino Unido.

Lott E.J., Bullock S.H. y Solís-Magallanes A. 1987. Floristic diversity and structure of upland and arroyo forests of coastal Jalisco. Biotropica 19:228-235.

Lu H.P., Wagner H.H. y Chen X.Y. 2007. A contribution diversity approach to evaluate species diversity. Basic and Applied Eco$\log y$ 8:1-12.

McCune B. y Mefford M.J. 1999. PC-ORD. Multivariate analysis for ecological data, versión 4.17. MjM Software Design, Gleneden Beach, Oregon, E.U.A.

Miranda F. y Hernández-X. E. 1963. Los tipos de vegetación de México y su clasificación. Boletín de la Sociedad Botánica de México 29:29-179.

Müllerried F. K. G. 1957. Geología de Chiapas. Gobierno Constitucional del Estado de Chiapas, Tuxtla Gutiérrez.
Müller-Dombois D. y Ellenberg H. 1974. Aims and methods of vegetation ecology. Wiley. Nueva York.

O’Brien E.M. 1993. Climate gradients in woody plant richness: towards an explanation based on an analysis of southern Africa's woody flora. Journal of Biogeography 20:181-198.

Oksanen J. 2010. Multivariate analysis of ecological communities in $\mathrm{R}$ : vegan tutorial. <cc.oulu.fi/ jarioksa/opetus/metodi/vegantutor.pdf $>$ (Consultado 21 de septiembre de 2010)

Palmer W.C. y Havens A.V. 1958. A graphical technique for determining evapotranpiration by the Thornthwaite method. Monthly Weather Review 86:123-128

Pennington R.T., Lavin M. y Oliveira-Filho A. 2009. Woody plant diversity, evolution, and ecology in the tropics: perspectives from seasonally dry tropical forests. Annual Review of Ecology, Evolution, and Systematics 40:437-457.

Pérez-García E.A., Meave J. y Gallardo C. 2001. Vegetación y flora de la región de Nizanda, Istmo de Tehuantepec, Oaxaca, México. Acta Botánica Mexicana 56:19-88.

Pérez-García E.A., Sevilha A.C., Meave J.A. y Scariot A. 2009. Floristic differentiation in limestone outcrops of southern Mexico and central Brazil: a beta diversity approach. Boletín de la Sociedad Botánica de México 84:45-58.

Pineda-García F., Arredondo-Amezcua L. y Ibarra-Manríquez G. 2007. Riqueza y diversidad de especies leñosas del bosque tropical caducifolio El Tarimo, Cuenca del Balsas, Guerrero. Revista Mexicana de Biodiversidad 78:129-139.

Quesada M., Sanchez-Azofeifa G.A., Alvarez-Añorve M., Stoner K.E., Avila-Cabadilla L., Calvo-Alvarado J., Castillo A., Espírito-Santo M.M., Fagundes M., Fernandes G.W., Gamonb J., Lopezaraiza-Mikel M., Lawrence D., Cerdeira Morellato L.D., Powers J.S., Neves F.S., Rosas-Guerrero V., Sayago R., Sanchez-Montoya G. 2009. Succession and management of tropical dry forests in the Americas: Review and new perspectives. Forest Ecology and Management 258:1014-1024.

$\mathrm{R}$ Development Core Team. 2010. A language and environment for statistical computing. Foundation for Statistical Computing, Vienna, Austria. <www.R-project.org> (Consultado 21 de septiembre de 2010)

Ramírez-Marcial N., Gonzalez-Espinosa M. y Williams-Linera G. 2001. Anthropogenic disturbance and tree diversity in Montane Rain Forests in Chiapas, Mexico. Forest Ecology and Management 154:311-326.

Ramírez-Marcial N., Camacho-Cruz A., González-Espinosa M. 2005. Potencial florístico para la restauración de bosques en Los Altos y Montañas del Norte de Chiapas. En González-Espinosa M., Ramírez-Marcial N., Ruiz Montoya L., eds. Diversidad biológica en Chiapas, pp. 325-369. Plaza y Valdés, México,

Ramírez-Marcial N., Camacho-Cruz A. y González-Espinosa M. 2008. Clasificación de grupos funcionales vegetales para la restauración del bosque mesófilo de montaña. En: Sánchez-Velázquez L.R., Galindo-González J. y Díaz-Fleischer F. Eds. Ecología, Manejo y conservación de los ecosistemas de montaña en México, pp. 51-72. Editorial Mundi-Prensa. México,

Reyes-García A. y Sousa M. 1997. Depresión Central de Chiapas, la Selva Baja Caducifolia. Listados Florísticos de México XVII. Instituto de Biología. Universidad Nacional Autónoma de México, México D.F.

Ricker M., Ramírez-Krauss I., Ibarra-Manríquez G., Martínez E., Ramos C.H., González-Medellín G., Gómez-Rodríguez G., Palacio-Prieto J.L. y Hernández H.M. 2007. Optimizing conser- 
vation of forest diversity: a country-wide approach in Mexico. Biodiversity and Conservation 16:1927-1957.

Ricklefs R.E. 1987. Community diversity: relative roles of local and regional processes. Science 235:167-171.

Rodríguez P. y Arita H. 2004. Beta diversity and latitude in North American mammals: testing the hypothesis of covariation. Ecography 27:547-556.

Rosenzweig M.L. 1968. Net primary productivity of terrestrial environments: predictions from climatological data. American Naturalist 102:67-84.

Rosenzweig M.L. 1995. Species diversity in space and time. Cambridge University Press. Reino Unido.

Rosenzweig M.L. y Abramsky Z. 1993. How are diversity and productivity related? En: Ricklefs R.E. y D. Schluter, Eds. Species diversity in ecological communities: historical and geographical perspectives, pp. 52-65. University of Chicago Press. EUA.

Rozza A.F., Turini-Farah F. y Riveiro-Rodrigues R. 2007. Ecological management of degraded forest fragments. En: Rodrigues R.R., Martins S.V. y Gandolfi S. Eds. High diversity forest restoration in degraded areas Methods and projects in Brazil, 171-
196. Nova Science Publishing, Nueva York.

Rzedowski J. 1978. Vegetación de México. Limusa. México, DF.

Salinas-Melgoza M.A. 2007. Patrones de variación de la diversidad de especies arbóreas con otras formas de crecimiento en las selvas bajas caducifolias de Colima. Tesis de Maestría. Centro de Investigaciones en Ecosistemas. Universidad Nacional Autónoma de México. Morelia, México.

Stephenson N.L. 1990. Climatic control of vegetation distribution: the role of the water balance. American Naturalist 135:649670 .

Trejo I. y Dirzo R. 2000. Deforestation of seasonally dry tropical forest: a national and local analysis in Mexico. Biological Conservation 94:133-142.

Trejo I. y Dirzo R. 2002. Floristic diversity of Mexican seasonally dry tropical forests. Biodiversity and Conservation 11:20632084.

Turc L. 1954. Le bilan d'eau des sols: relations entre les precipitation, l'évaporation et l'écoulement. Annales Agronomiques 5:491-596.

Wright D.H. 1983. Species-energy theory: an extension of speciesarea theory. Oikos 41:496-506.

Recibido: 10 de mayo de 2010

Aceptado: $1^{\circ}$ de noviembre de 2010 
Apéndice 1. Relación taxonómica y promedio de los valores de importancia relativa por asociación vegetal (Mat.opo-Ter.tep = Matayba-oppositifolia-Ternstroemia tepezapote, Bur.sim-Coc.vit = Bursera simaruba-Cochlospermum vitiifolium, Leu.div-Cei.aes = Leucaena diversifolia-Ceiba aesculifolia, Que.ped-Pin.ooc = Quercus peduncularis- Pinus oocarpa y Que.pol-Que.seg = Quercus polymorpha-Quercus segoviensis) de las 233 especies registradas en la Depresión Central de Chiapas. La afinidad sucesional: $\mathrm{P}=$ Pionera, $\mathrm{Si}=$ Secundaria inicial, $\mathrm{T}=$ Tardía, ND = No determinado.

\begin{tabular}{|c|c|c|c|c|c|c|c|}
\hline \multirow[b]{2}{*}{ Familia } & \multirow[b]{2}{*}{ Nombre científico } & \multirow[b]{2}{*}{$\begin{array}{r}\text { Afinidad } \\
\text { sucesional }\end{array}$} & \multicolumn{5}{|c|}{ Asociación } \\
\hline & & & $\begin{array}{l}\text { Mat.opo- } \\
\text { Ter.tep }\end{array}$ & $\begin{array}{c}\text { Bur.sim- } \\
\text { Coc.vit }\end{array}$ & $\begin{array}{l}\text { Leu.div- } \\
\text { Cei.aes }\end{array}$ & $\begin{array}{l}\text { Que.ped- } \\
\text { Pin.ooc }\end{array}$ & $\begin{array}{l}\text { Que.pol- } \\
\text { Que.seg }\end{array}$ \\
\hline Agavaceae & Yucca guatemalensis Baker & $\mathrm{T}$ & & 11.9 & & & \\
\hline Anacardiaceae & Astronium graveolens Jacq. & $\mathrm{T}$ & & 4.3 & 4.3 & & \\
\hline Anacardiaceae & Comocladia guatemalensis Donn. Sm. & $\mathrm{Si}$ & & 7.7 & 3.8 & & \\
\hline Anacardiaceae & Mosquitoxylum jamaicense Krug \& Urb. & Si & 3.8 & & & & \\
\hline Anacardiaceae & Pistacia mexicana Kunth & $\mathrm{Si}$ & & & & 6.7 & 7.0 \\
\hline Anacardiaceae & Rhus schiedeana Schltdl. & $\mathrm{Si}$ & & & & & 4.3 \\
\hline Anacardiaceae & Spondias purpurea L. & $\mathrm{Si}$ & & 3.4 & 3.0 & & \\
\hline Anacardiaceae & Tapirira mexicana Marchand & $\mathrm{T}$ & 6.2 & & & & \\
\hline Annonaceae & Annona cherimola Mill. & $\mathrm{Si}$ & & 2.4 & 6.1 & & \\
\hline Annonaceae & Annona liebmanniana Baill. & $\mathrm{T}$ & & 1.4 & & & \\
\hline Annonaceae & Especie 109 & $\mathrm{~T}$ & & 1.4 & 1.5 & & \\
\hline Annonaceae & Rollinia membranacea Triana \& Planch. & $\mathrm{Si}$ & 10.2 & 1.1 & & & \\
\hline Apocynaceae & Cascabela ovata (Cav. ) Lippold & Si & & 2.8 & 1.4 & & \\
\hline Apocynaceae & Plumeria rubra L. & $\mathrm{Si}$ & & 4.3 & 0.9 & & \\
\hline Apocynaceae & Stemmadenia obovata (Benth.) Woodson & $P$ & & 4.9 & 3.7 & & \\
\hline Apocynaceae & Tabernaemontana amygdalifolia Jacq. & $P$ & 1.1 & & & & \\
\hline Araliaceae & Dendropanax arboreus (L.) Decne. \& Planch. & $\mathrm{T}$ & 3.3 & & & & \\
\hline Araliaceae & Oreopanax capitatus (Jacq.) Decne. \& Planch. & $\mathrm{Si}$ & & & & & 6.1 \\
\hline Araliaceae & Oreopanax geminatus Marchal & $\mathrm{Si}$ & 4.0 & 1.3 & & & \\
\hline Asteraceae & Clibadium arboreum Donn. Sm. & $P$ & & 2.6 & 2.0 & & \\
\hline Asteraceae & Critoniopsis leiocarpa (DC.) H. Rob. & $\mathrm{P}$ & & & & & 3.7 \\
\hline Asteraceae & Perymenium grande Hemsl. & Si & & & & & 2.1 \\
\hline Asteraceae & Sinclairia discolor Hook. \&Arn. & $P$ & & & & 9.3 & \\
\hline Bignoniaceae & Tabebuia chrysantha G. Nicholson & Si & & 4.4 & & & \\
\hline Bignoniaceae & Tabebuia rosea (Bertol.) A. DC. & Si & & 5.4 & 4.3 & & \\
\hline Bignoniaceae & Tecoma stans (L.) Juss. ex Kunth & $\mathrm{P}$ & 1.1 & 4.5 & & & \\
\hline Bixaceae & Cochlospermum vitifolium (Willd.) Spreng. & $\mathrm{P}$ & & 7.1 & 7.1 & 2.0 & \\
\hline Boraginaceae & Cordia alliodora (Ruiz \& Pav.) Oken & $\mathrm{Si}$ & & 1.6 & 1.7 & & \\
\hline Boraginaceae & Cordia foliosa M. Martens \& Galeotti & $\mathrm{T}$ & & 5.7 & 2.5 & & \\
\hline Boraginaceae & Cordia stellifera I.M. Johnst. & $\mathrm{T}$ & & 4.4 & 18.9 & & \\
\hline Boraginaceae & Ehretia latifolia DC. & $\mathrm{P}$ & & 1.4 & 0.7 & & \\
\hline Boraginaceae & Ehretia sp146 & $\mathrm{P}$ & & 2.0 & & & \\
\hline Burseraceae & Bursera ariensis (Kunth) McVaugh \& Rzed. & $\mathrm{T}$ & & 2.6 & & & \\
\hline Burseraceae & Bursera bipinnata (DC.) Engl. & Si & & 21.8 & & 12.3 & \\
\hline Burseraceae & Bursera excelsa (Kunth) Engl. & $\mathrm{Si}$ & & 6.4 & 3.2 & 6.5 & 2.0 \\
\hline Burseraceae & Bursera heteresthes Bullock & $\mathrm{T}$ & & 3.7 & 3.3 & & \\
\hline Burseraceae & Bursera simaruba (L.) Sarg. & $\mathrm{P}$ & 6.4 & 18.1 & 13.8 & 7.3 & \\
\hline Burseraceae & Protium copal (Schltdl. \& Cham.) Engl. & $\mathrm{T}$ & 5.3 & & & & \\
\hline Cactaceae & Opuntia dejecta Salm-Dyck & $\mathrm{T}$ & & 3.6 & 5.6 & & \\
\hline Cannabaceae & Trema micrantha (L.) Blume & $\mathrm{P}$ & & 3.5 & & & \\
\hline Capparaceae & Capparidastrum baducca (L.) Hutch. & $\mathrm{T}$ & & 2.1 & & & \\
\hline Caricaceae & Carica cauliflora Jacq. & $\mathrm{Si}$ & & 1.8 & & & \\
\hline Caricaceae & Jacaratia mexicana A. DC. & $P$ & & 8.6 & & & \\
\hline Celastraceae & Crossopetalum tonduzii (Loes.) Lundell & $\mathrm{T}$ & 15.4 & & & & \\
\hline
\end{tabular}


Apéndice 1. Continuación

\begin{tabular}{|c|c|c|c|c|c|c|c|}
\hline \multirow[b]{2}{*}{ Familia } & \multirow[b]{2}{*}{ Nombre científico } & \multirow[b]{2}{*}{$\begin{array}{r}\text { Afinidad } \\
\text { sucesional }\end{array}$} & \multicolumn{5}{|c|}{ Asociación } \\
\hline & & & $\begin{array}{l}\text { Mat.opo- } \\
\text { Ter.tep }\end{array}$ & $\begin{array}{l}\text { Bur.sim- } \\
\text { Coc.vit }\end{array}$ & $\begin{array}{l}\text { Leu.div- } \\
\text { Cei.aes }\end{array}$ & $\begin{array}{l}\text { Que.ped- } \\
\text { Pin.ooc }\end{array}$ & $\begin{array}{l}\text { Que.pol- } \\
\text { Que.seg }\end{array}$ \\
\hline Celastraceae & Maytenus schippii Lundell & $\mathrm{T}$ & & 3.9 & 3.4 & & \\
\hline Celastraceae & Schaefferia sp236 & $\mathrm{T}$ & & 1.8 & & & \\
\hline Celastraceae & Semialarium mexicanum (Miers) Mennega & $\mathrm{Si}$ & 3.0 & 5.0 & 3.9 & 1.8 & \\
\hline Celastraceae & Wimmeria bartlettii Lundell & $\mathrm{T}$ & 2.4 & 4.1 & & & \\
\hline Celastraceae & Wimmeria pubescens Radlk. & $\mathrm{T}$ & & 9.2 & 1.4 & & \\
\hline Chrysobalanaceae & Licania arborea Seem. & $\mathrm{T}$ & & 1.3 & 5.5 & & \\
\hline Clusiaceae & Clusia rosea Jacq. & $\mathrm{T}$ & & 0.9 & & & \\
\hline Clusiaceae & Clusia salvinii Donn. Sm. & $\mathrm{T}$ & & 5.8 & & & \\
\hline Combretaceae & Bucida macrostachya Standl. & $\mathrm{T}$ & & 8.5 & & 1.9 & \\
\hline Combretaceae & Terminalia oblonga (Ruiz \& Pav.) Steud. & $\mathrm{T}$ & 2.1 & 11.0 & & & \\
\hline Dilleniaceae & Curatella americana L. & $\mathrm{Si}$ & & 2.2 & & & \\
\hline Ebenaceae & Diospyros salicifolia Humb. \& Bonpl. ex Willd. & $\mathrm{T}$ & & 2.7 & & & \\
\hline Ericaceae & Arbutus xalapensis Kunth & $\mathrm{T}$ & & & & & 1.0 \\
\hline Erythroxylaceae & Erythroxylum rotundifolium Lunan & $\mathrm{T}$ & & & 3.3 & & \\
\hline Erythroxylaceae & Erythroxylum tabascense Britton & $\mathrm{T}$ & 0.9 & & & & \\
\hline Euphorbiaceae & Alchornea chiapasana Miranda & $\mathrm{Si}$ & 5.6 & & & & \\
\hline Euphorbiaceae & Alchornea latifolia Sw. & $\mathrm{P}$ & 3.2 & & & & \\
\hline Euphorbiaceae & $\begin{array}{l}\text { Bernardia dodecandra (Sessé ex Cavagnaro) } \\
\text { McVaugh }\end{array}$ & $\mathrm{T}$ & 6.5 & 1.7 & 2.4 & & \\
\hline Euphorbiaceae & Cnidoscolus multilobus (Pax) I.M. Johnst. & $P$ & & 2.0 & 3.6 & & \\
\hline Euphorbiaceae & $\begin{array}{l}\text { Croton carpostellatus B. L. Leon \& } \\
\text { Martínez-Gordillo }\end{array}$ & $\mathrm{T}$ & 0.7 & & & & \\
\hline Euphorbiaceae & Croton glabellus L. & $\mathrm{T}$ & 3.5 & & & & \\
\hline Euphorbiaceae & Croton reflexifolius Kunth & $\mathrm{Si}$ & 4.9 & & & & \\
\hline Euphorbiaceae & Croton sp101 & $\mathrm{T}$ & & 2.2 & & & \\
\hline Euphorbiaceae & Croton sp181 & $\mathrm{T}$ & & 3.7 & & & \\
\hline Euphorbiaceae & Euphorbia schlechtendalii Bois. & $\mathrm{T}$ & & 2.7 & 0.7 & & \\
\hline Euphorbiaceae & Hura polyandra Baill. & $\mathrm{T}$ & & 25.1 & & & \\
\hline Euphorbiaceae & Jatropha curcas L. & $\mathrm{P}$ & & 5.3 & 15.9 & & \\
\hline Euphorbiaceae & Sebastiania cruenta (Standl. \& Steyer.) Miranda. & $\mathrm{T}$ & & & & & 2.7 \\
\hline Euphorbiaceae & Sebastiania sp55 & $\mathrm{T}$ & 5.8 & & & & \\
\hline Fabaceae & Acacia cornigera (L.) Wild & $\mathrm{P}$ & & 4.5 & 0.7 & 2.0 & \\
\hline Fabaceae & Acacia pennatula (Schltdl. \& Cham.) Benth. & $P$ & & 5.3 & 17.1 & 10.6 & 4.1 \\
\hline Fabaceae & Acosmium panamense (Benth.) Yakovlev & $\mathrm{T}$ & 3.4 & & & & \\
\hline Fabaceae & Albizia lebbeck (L.) Benth. & $\mathrm{Si}$ & & 5.6 & & & \\
\hline Fabaceae & Andira inermis (W. Wright) Kunth ex DC. & $\mathrm{T}$ & & 1.3 & 5.7 & & \\
\hline Fabaceae & Bauhinia divaricata L. & $\mathrm{P}$ & & 5.9 & & & \\
\hline Fabaceae & Bauhinia ungulata L. & $\mathrm{P}$ & & 2.5 & 5.9 & & \\
\hline Fabaceae & Caesalpinia eriostachys Benth. & Si & & 21.2 & & & \\
\hline Fabaceae & Cassia sp167 & $\mathrm{Si}$ & & 1.8 & & & \\
\hline Fabaceae & Dalbergia glabra (Mill.) Standl. & $\mathrm{T}$ & & 1.1 & 9.8 & 6.6 & \\
\hline Fabaceae & Diphysa americana (Mill.) M. Sousa & $\mathrm{T}$ & & 12.5 & & 4.8 & \\
\hline Fabaceae & Enterolobium cyclocarpum (Jacq.) Griseb. & Si & & 21.5 & 3.1 & & \\
\hline Fabaceae & Erythrina chiapasana Krukoff & Si & & & & 29.1 & 7.7 \\
\hline Fabaceae & Erythrina folkersii Krukoff \& Moldenke & Si & & 3.3 & 20.1 & 3.7 & 1.3 \\
\hline Fabaceae & Gliricidia sepium (Jacq.) Kunth ex Walp. & $\mathrm{P}$ & & 2.7 & & & \\
\hline Fabaceae & Haematoxylum campechianum L. & $\mathrm{T}$ & & 1.5 & & & \\
\hline Fabaceae & Hymenaea courbaril L. & $\mathrm{T}$ & & 13.2 & & & \\
\hline
\end{tabular}


Apéndice 1. Continuación

\begin{tabular}{|c|c|c|c|c|c|c|c|}
\hline \multirow[b]{2}{*}{ Familia } & \multirow[b]{2}{*}{ Nombre científico } & \multirow[b]{2}{*}{$\begin{array}{r}\text { Afinidad } \\
\text { sucesional }\end{array}$} & \multicolumn{5}{|c|}{ Asociación } \\
\hline & & & $\begin{array}{l}\text { Mat.opo- } \\
\text { Ter.tep }\end{array}$ & $\begin{array}{l}\text { Bur.sim- } \\
\text { Coc.vit }\end{array}$ & $\begin{array}{l}\text { Leu.div- } \\
\text { Cei.aes }\end{array}$ & $\begin{array}{l}\text { Que.ped- } \\
\text { Pin.ooc }\end{array}$ & $\begin{array}{l}\text { Que.pol- } \\
\text { Que.seg }\end{array}$ \\
\hline Fabaceae & Inga fagifolia (L.) Wild. ex Benth. & $\mathrm{Si}$ & & 2.9 & & & \\
\hline Fabaceae & Leucaena collinsii Britton \& Rose & $\mathrm{P}$ & & 4.9 & 0.9 & 4.6 & 5.2 \\
\hline Fabaceae & Leucaena diversifolia (Schltdl.) Benth. & $\mathrm{P}$ & & 11.1 & 26.4 & 7.3 & 4.5 \\
\hline Fabaceae & Leucaena esculenta (Moc. \& Sessé ex DC.) Be & า. $P$ & & & & & 1.1 \\
\hline Fabaceae & Leucaena shannonii Donn. Sm. & $\mathrm{Si}$ & 1.1 & 8.8 & 3.5 & 1.5 & \\
\hline Fabaceae & Lonchocarpus hondurensis Benth. & $\mathrm{Si}$ & & & & 2.2 & \\
\hline Fabaceae & Lonchocarpus rugosus Benth. & $\mathrm{Si}$ & & 1.3 & 6.1 & 2.9 & 4.8 \\
\hline Fabaceae & Lysiloma acapulcense (Kunth) Benth. & $P$ & & & & & 1.9 \\
\hline Fabaceae & Lysiloma divaricatum (Jacq.) J.F. Macbr. & $P$ & & 11.9 & & & \\
\hline Fabaceae & Machaerium chiapense Brandegee & $\mathrm{T}$ & & 1.5 & & 3.5 & \\
\hline Fabaceae & Machaerium cirrhiferum Pittier & $\mathrm{T}$ & & 19.3 & & & \\
\hline Fabaceae & Mimosa tenuiflora (Willd.) Poir. & $\mathrm{P}$ & & & 5.9 & & \\
\hline Fabaceae & Platymiscium yucatanum Standl. & $\mathrm{T}$ & & & 2.7 & & \\
\hline Fabaceae & Poeppigia procera C. Presl & $\mathrm{P}$ & & 17.9 & 4.0 & & \\
\hline Fabaceae & Senegalia picachensis Britton \& Rose & $\mathrm{Si}$ & & 8.2 & 12.14 .0 & & \\
\hline Fabaceae & Senna atomaria (L.) H.S. Irwin \& Barneby & $\mathrm{P}$ & & 3.8 & 1.4 & & \\
\hline Fagaceae & Quercus acutifolia Née & Si & & & & 38.3 & \\
\hline Fagaceae & Quercus castanea Née & $\mathrm{Si}$ & & & & 5.2 & \\
\hline Fagaceae & Quercus conspersa Benth. & $\mathrm{Si}$ & & & & 17.7 & \\
\hline Fagaceae & Quercus crispipilis Trel. & $\mathrm{Si}$ & & & & & 6.1 \\
\hline Fagaceae & Quercus peduncularis Née & $\mathrm{Si}$ & & 24.2 & & 59.0 & 12.9 \\
\hline Fagaceae & Quercus polymorpha Schltdl. \& Cham. & Si & & & & 15.4 & 65.6 \\
\hline Fagaceae & Quercus sapotifolia Liebm. & $\mathrm{Si}$ & & & & & 14.9 \\
\hline Fagaceae & Quercus segoviensis Liebm. & Si & & & & & 57.7 \\
\hline Garryaceae & Garrya laurifolia Hartw. ex Benth. & $\mathrm{Si}$ & & & & & 4.5 \\
\hline Hernandiaceae & Gyrocarpus mocinoi Espejo & $\mathrm{T}$ & & 2.2 & 0.8 & & \\
\hline Lauraceae & Cinnamomum sp33 & $\mathrm{T}$ & 1.7 & & & & 1.3 \\
\hline Lauraceae & Licaria caudata (Lundell) Kosterm. & $\mathrm{T}$ & 2.7 & & 1.6 & & \\
\hline Lauraceae & Nectandra reticulata (Ruiz \& Pav.) Mez & $\mathrm{T}$ & 1.4 & & & & \\
\hline Lauraceae & Nectandra salicifolia (Kunth) Nees & $\mathrm{T}$ & 4.7 & 0.9 & 2.7 & & \\
\hline Lauraceae & Persea liebmannii Mez & $\mathrm{T}$ & 3.0 & & & & \\
\hline Lauraceae & Persea schiedeana Nees & $\mathrm{T}$ & & & & 2.7 & \\
\hline Malpighiaceae & Byrsonima crassifolia (L.) Kunth & $\mathrm{Si}$ & & 2.1 & & 4.8 & \\
\hline Malpighiaceae & Heteropteris sp87 & $\mathrm{T}$ & & 4.9 & & & \\
\hline Malpighiaceae & Malpighia glabra L. & $\mathrm{Si}$ & & 1.3 & & & \\
\hline Malvaceae & Ceiba acuminata (S. Watson) Rose & $\mathrm{T}$ & & 3.9 & & & \\
\hline Malvaceae & Ceiba aesculifolia (Kunth) Britten \& Baker f. & $\mathrm{Si}$ & & 3.8 & 21.0 & & \\
\hline Malvaceae & Ceiba pentandra (L.) Gaertn. & Si & & 2.8 & & & \\
\hline Malvaceae & Ceiba sp190 & $\mathrm{Si}$ & & 6.8 & & & \\
\hline Malvaceae & Guazuma ulmifolia Lam. & $P$ & & 3.9 & 24.5 & 5.2 & \\
\hline Malvaceae & Hampea stipitata S. Watson & $\mathrm{P}$ & 1.8 & & & & \\
\hline Malvaceae & Heliocarpus terebinthinaceus (DC.) Hochr. & $\mathrm{P}$ & & 11.9 & 2.3 & 8.6 & \\
\hline Malvaceae & Luehea candida (Moc. \& Sessé ex DC.) Mart. & Si & & 4.9 & 2.1 & & \\
\hline Malvaceae & Luehea speciosa Willd. & Si & & 8.3 & & & \\
\hline Malvaceae & Pseudobombax ellipticum (Kunth) Dugand & Si & & 9.1 & 19.1 & 4.4 & \\
\hline Malvaceae & Trichospermum mexicanum (DC.) Baill. & $\mathrm{P}$ & 8.4 & & & & \\
\hline Meliaceae & Cedrela odorata L. & $\mathrm{Si}$ & 4.3 & 12.2 & & & \\
\hline Meliaceae & Cedrela salvadorensis Standl. & $\mathrm{T}$ & & 4.2 & 2.3 & & \\
\hline
\end{tabular}


Apéndice 1. Continuación

\begin{tabular}{|c|c|c|c|c|c|c|c|}
\hline \multirow[b]{2}{*}{ Familia } & \multirow[b]{2}{*}{ Nombre científico } & \multirow{2}{*}{$\begin{array}{r}\text { Afinidad } \\
\text { sucesional }\end{array}$} & \multicolumn{5}{|c|}{ Asociación } \\
\hline & & & $\begin{array}{l}\text { Mat.opo- } \\
\text { Ter.tep }\end{array}$ & $\begin{array}{l}\text { Bur.sim- } \\
\text { Coc.vit }\end{array}$ & $\begin{array}{l}\text { Leu.div- } \\
\text { Cei.aes }\end{array}$ & $\begin{array}{l}\text { Que.ped- } \\
\text { Pin.ooc }\end{array}$ & $\begin{array}{l}\text { Que.pol- } \\
\text { Que.seg }\end{array}$ \\
\hline Meliaceae & Swietenia humilis Zucc. & $\mathrm{T}$ & & 2.6 & & & \\
\hline Meliaceae & Trichilia hirta L. & $\mathrm{Si}$ & & 7.2 & 8.5 & & \\
\hline Meliaceae & Trichilia martiana A. DC. & $\mathrm{Si}$ & & 4.6 & & & \\
\hline Menispermaceae & Abuta panamensis (Standl.) Krukoff \& Barneby & $\mathrm{T}$ & & 4.5 & & 0.8 & \\
\hline Moraceae & Brosimum alicastrum Sw. & $\mathrm{T}$ & & 1.1 & 13.9 & & \\
\hline Moraceae & Ficus aurea Nutt. & $\mathrm{Si}$ & 4.2 & 9.2 & & & 8.5 \\
\hline Moraceae & Ficus colubrinae Standl. & Si & 3.7 & & & & \\
\hline Moraceae & Ficus pertusa L.f. & $\mathrm{Si}$ & 6.2 & 2.8 & 3.7 & & \\
\hline Moraceae & Ficus yoponensis Desv. & $\mathrm{Si}$ & 4.5 & & 4.9 & & \\
\hline Moraceae & Maclura tinctoria (L.) D. Don ex Steud. & $\mathrm{T}$ & & 1.8 & 3.6 & & \\
\hline Moraceae & Pseudolmedia spuria (Sw.) Griseb. & $\mathrm{T}$ & 5.2 & 2.5 & & & \\
\hline Myrtaceae & Eugenia acapulcensis Steud. & $\mathrm{T}$ & & 4.6 & & & 5.4 \\
\hline Myrtaceae & Eugenia capulioides Lundell & $\mathrm{T}$ & & 2.4 & & & 7.0 \\
\hline Myrtaceae & Eugenia oerstediana O. Berg. & $\mathrm{T}$ & 3.3 & & & & \\
\hline Myrtaceae & Eugenia origanoides O. Berg. & $\mathrm{T}$ & 0.7 & 1.1 & & & \\
\hline Myrtaceae & Myrcianthes fragrans (Sw.) McVaugh & $\mathrm{T}$ & 1.2 & & & & \\
\hline Myrtaceae & Pimenta dioica (L.) Merr. & $\mathrm{T}$ & 0.9 & & & & \\
\hline Oleaceae & Fraxinus purpusii Brandegee & $\mathrm{Si}$ & & 3.6 & & & 9.7 \\
\hline Olacaceae & Schoepfia flexuosa (Ruiz \& Pav.) Schult. & $\mathrm{T}$ & & & & & 7.3 \\
\hline Onagraceae & Hauya elegans DC. & $\mathrm{Si}$ & & 5.4 & & & 7.2 \\
\hline Onagraceae & Hauya heydeana Donn. Sm. & Si & & 5.6 & & & \\
\hline Pentaphylacaceae & Ternstroemia tepezapote Schltdl. \& Cham. & $\mathrm{Si}$ & 14.5 & & & & 8.4 \\
\hline Phyllanthaceae & Phyllanthus acuminatus Vahl & $\mathrm{T}$ & & 3.0 & & & \\
\hline Picramniaceae & Alvaradoa amorphoides Liebm. & Si & & 8.2 & 7.3 & & 1.9 \\
\hline Picramniaceae & Picramnia antidesma Sw. & $\mathrm{T}$ & 0.7 & & & & \\
\hline Pinaceae & Pinus maximinoi H.E. Moore & $\mathrm{P}$ & & & & 9.7 & \\
\hline Pinaceae & Pinus oocarpa Schiede ex Schltdl. & $P$ & & & & 36.9 & \\
\hline Piperaceae & Piper diandrum C. DC. & $\mathrm{Si}$ & 1.3 & & & & \\
\hline Polygonaceae & Coccoloba acapulcensis Standl. & $\mathrm{T}$ & & 5.1 & & & \\
\hline Polygonaceae & Coccoloba floribunda (Benth.) Lindau & $\mathrm{T}$ & 12.4 & 4.5 & 5.1 & & \\
\hline Primulaceae & Ardisia brevis Lundell & $\mathrm{T}$ & 0.8 & & & & \\
\hline Primulaceae & Myrsine coriacea (Sw.) R. Br. ex Roem. \& Schult. & $\mathrm{Si}$ & & & & & 2.5 \\
\hline Rhamnaceae & Colubrina arborescens (Mill.) Sarg. & $\mathrm{T}$ & & 1.9 & & & \\
\hline Rhamnaceae & Colubrina guatemalensis Standl. & $\mathrm{T}$ & & 2.2 & & & \\
\hline Rhamnaceae & Rhamnus serrata Humb. \& Bonpl. ex Willd. & $\mathrm{T}$ & & 1.3 & & & \\
\hline Rosaceae & Prunus brachybotrya Zucc. & $\mathrm{T}$ & & & & 2.3 & \\
\hline Rosaceae & Prunus lundelliana Standl. & $\mathrm{T}$ & & & & & 5.4 \\
\hline Rosaceae & Prunus serotina Ehrh. & $\mathrm{P}$ & & & & & 1.7 \\
\hline Rubiaceae & Alibertia edulis (Rich.) A. Rich. ex DC. & Si & & 3.3 & & & \\
\hline Rubiaceae & Calycophyllum candidissimum (Vahl) DC. & $\mathrm{T}$ & & 18.1 & 4.8 & & \\
\hline Rubiaceae & Chiococca alba (L.) Hitchc. & $\mathrm{Si}$ & & & & & 2.5 \\
\hline Rubiaceae & Exostema mexicanum A. Gray & $\mathrm{T}$ & & 14.2 & & & \\
\hline Rubiaceae & Genipa americana L. & $\mathrm{T}$ & & 15.5 & 1.3 & & \\
\hline Rubiaceae & Guettarda combsii Urb. & $\mathrm{Si}$ & 6.4 & & 2.3 & & \\
\hline Rubiaceae & Psychotria limonensis K. Krause & $\mathrm{Si}$ & 0.9 & & & & \\
\hline Rubiaceae & Randia armata (Sw.) DC. & $\mathrm{Si}$ & & 2.3 & 3.8 & & \\
\hline Rubiaceae & Randia capitata DC. & $\mathrm{T}$ & & 1.6 & 1.7 & & \\
\hline Rubiaceae & Solenandra mexicana (A. Gray) Borhidi & $\mathrm{T}$ & & 4.8 & 5.9 & & \\
\hline
\end{tabular}


Apéndice 1. Continuación

\begin{tabular}{|c|c|c|c|c|c|c|c|}
\hline \multirow[b]{2}{*}{ Familia } & \multirow[b]{2}{*}{ Nombre científico } & \multirow[b]{2}{*}{$\begin{array}{r}\text { Afinidad } \\
\text { sucesional }\end{array}$} & \multicolumn{5}{|c|}{ Asociación } \\
\hline & & & $\begin{array}{l}\text { Mat.opo- } \\
\text { Ter.tep }\end{array}$ & $\begin{array}{l}\text { Bur.sim- } \\
\text { Coc.vit }\end{array}$ & $\begin{array}{l}\text { Leu.div- } \\
\text { Cei.aes }\end{array}$ & $\begin{array}{l}\text { Que.ped- } \\
\text { Pin.ooc }\end{array}$ & $\begin{array}{l}\text { Que.pol- } \\
\text { Que.seg }\end{array}$ \\
\hline Rutaceae & Amyris elemifera $\mathrm{L}$. & $\mathrm{T}$ & & & & & 2.9 \\
\hline Rutaceae & Zanthoxylum aguilarii Standl. \& Steyerm. & $\mathrm{T}$ & & 3.1 & & & 0.9 \\
\hline Rutaceae & Zanthoxylum caribaeum Lam. & $\mathrm{T}$ & & 4.2 & 1.7 & & \\
\hline Rutaceae & Zanthoxylum riedelianum Engl. & $\mathrm{T}$ & 2.9 & 0.8 & & & \\
\hline Salicaceae & Casearia nitida (L.) Jacq. & $\mathrm{P}$ & & 1.9 & 3.4 & & \\
\hline Salicaceae & Laetia thamnia L. & $\mathrm{T}$ & 4.2 & & & & \\
\hline Salicaceae & Xylosma flexuosa (Kunth) Hemsl. & $\mathrm{T}$ & & & & & 1.0 \\
\hline Salicaceae & Xylosma panamensis Turcz. & $\mathrm{T}$ & & 1.7 & & 1.1 & \\
\hline Salicaceae & Xylosma velutina (Tul.) Triana \& Planch. & $\mathrm{T}$ & & 6.5 & & & \\
\hline Salicaceae & Zuelania guidonia (Sw.) Britton \& Millsp. & $\mathrm{Si}$ & 23.6 & 7.6 & 2.5 & & \\
\hline Sapindaceae & Allophylus camptostachys Radlk. & $\mathrm{T}$ & 1.2 & 6.1 & & & \\
\hline Sapindaceae & Matayba oppositifolia (A. Rich.) Britton & $\mathrm{T}$ & 14.6 & 2.4 & & & \\
\hline Sapindaceae & Sapindus saponaria L. & $\mathrm{Si}$ & & & & 1.1 & \\
\hline Sapotaceae & Chrysophyllum mexicanum Brandegee ex Standl. & $\mathrm{Si}$ & & & 3.9 & & \\
\hline Sapotaceae & Manilkara zapota (L.) P. Royen & $\mathrm{T}$ & & & 5.4 & & \\
\hline Sapotaceae & Sideroxylon capiri (A. DC.) Pittier & $\mathrm{T}$ & & 4.4 & 2.4 & & \\
\hline Sapotaceae & Sideroxylon celastrinum (Kunth) T.D. Penn. & $\mathrm{T}$ & & 3.7 & & & \\
\hline Sapotaceae & Sideroxylon salicifolium (L.) Lamb. & $\mathrm{T}$ & & 10.9 & 4.2 & & \\
\hline Theophrastaceae & Jacquinia macrocarpa Cav. var. macrocarpa & $\mathrm{T}$ & & 5.1 & 2.1 & & \\
\hline Thymelaeaceae & Daphnopsis americana (Mill.) J.R. Johnst. & Si & & 7.4 & & & \\
\hline Urticaceae & Cecropia obtusifolia Bertol. & $P$ & 2.1 & & 2.7 & & \\
\hline Urticaceae & Coussapoa sp38 & $\mathrm{T}$ & 9.2 & & & & \\
\hline Urticaceae & Myriocarpa longipes Liebm. & Si & & 3.4 & & & \\
\hline Urticaceae & Urera baccifera (L.) Gaudich. ex Wedd. & $\mathrm{Si}$ & & 8.0 & & & \\
\hline Verbenaceae & Citharexylum hexangulare Greenm. & $\mathrm{T}$ & 0.7 & & & & \\
\hline Verbenaceae & Lippia myriocephala Schltdl. \& Cham. & $\mathrm{P}$ & 4.9 & & & & \\
\hline Verbenaceae & Petrea volubilis $\mathrm{L}$. & $\mathrm{T}$ & & 4.6 & & & \\
\hline Violaceae & $\begin{array}{l}\text { Orthion malpighiifolium (Standl.) Standl. } \\
\text { \& Steyerm. }\end{array}$ & $\mathrm{T}$ & 7.4 & & & & \\
\hline Zamiaceae & Dioon merolae De Luca, Sabato \& Vázq. Torres & $\mathrm{T}$ & & & 2.1 & & \\
\hline ND & especie sp127 & ND & & & 2.3 & & \\
\hline ND & especie sp129 & ND & & & 1.8 & & \\
\hline ND & especie sp140 & ND & & 5.9 & & & \\
\hline ND & especie sp164 & ND & & 3.7 & & & \\
\hline ND & especie sp191 & ND & & 2.8 & & & \\
\hline ND & especie sp196 & ND & & 3.3 & & & \\
\hline ND & especie sp201 & ND & & 8.4 & & & \\
\hline ND & especie sp203 & ND & & 2.8 & & & \\
\hline ND & especie sp207 & ND & & 5.0 & & & \\
\hline ND & especie sp216 & ND & & 1.4 & 3.6 & & \\
\hline ND & especie sp220 & ND & & 5.0 & & & \\
\hline ND & especie sp222 & ND & & 1.5 & & & \\
\hline ND & especie sp223 & ND & & 1.1 & & & \\
\hline ND & especie sp224 & ND & & 9.0 & & & \\
\hline ND & especie sp225 & ND & & 2.0 & & & \\
\hline ND & especie sp227 & ND & & 3.4 & & & \\
\hline ND & especie sp239 & ND & & & & & 0.8 \\
\hline ND & especie sp277 & ND & & & & 2.7 & \\
\hline
\end{tabular}

